\title{
Health-related care for the Neanderthal Shanidar 1
}

\author{
LAURA KENT
}

\section{Abstract}

The bioarchaeology of care methodology is used to identify health-related care for prehistoric hominids using the skeletal indications of survival with a disability or debilitating disease that would have resulted in death if care was not given. This model involves four stages and was applied to the Neanderthal Shanidar 1 in order to evaluate the type of care possibly received by the individual and what this caregiving behaviour suggests about Neanderthal culture and behaviour. The skeletal remains of Shanidar 1 represents an adult male of advanced age who suffered from a number of debilitating pathologies that would have affected his ability to survive and contribute to his social group. Shanidar 1 required health-related care in the form of direct support and accommodation of a different role within the social group in order to survive to his age at death. The survival of Shanidar 1 to old age implies Neanderthals were capable of changing their behaviour in order to care for and accommodate injured members of their social group. This evidence of health-related care for Shanidar 1 suggests Neanderthals had a greater level of behavioural flexibility and social complexity than previously believed.

\section{Introduction}

The bioarchaeology of care methodology was developed by Lorna Tilley to identify health-related care in the past using physical indicators of long-term survival with a disability or disease (Tilley, 2012). Health-related care in the past can be inferred from examining the skeletal remains of individuals from prehistoric cultures that show evidence of survival with a deliberating disease, injury or disability that, without care and support, would have killed the individual prior to reaching their age at death (Tilley \& Oxenham, 2011). The examination of health-related care can be used to interpret the behaviour, beliefs, values and social practices of past societies and cultures (Tilley, 2015). Health-related care refers to the act of deliberately deciding to deliver assistance to an individual suffering from a disease, injury or disability (Tilley, 2015). Health-related care differs between societies and is related to the cultural beliefs, practices, knowledge, traditions, resources and social organisation 
of the society (Tilley \& Oxenham, 2011). Care is either provided temporarily or over a long period of time, depending on the needs of the individual, and can be in the form of direct support or accommodation. Direct-support caregiving involves practical assistance and nursing necessary for the individual's survival, such as providing food or water, helping with movement, maintaining hygiene and protecting the individual from potential dangers (Tilley, 2015). Accommodationrelated caregiving involves the adjustment of expectations, practices or behaviour to enable a disabled individual to participate in society (Tilley, 2015).

Health-related care can be examined using four stages to infer the type of care received by an individual and interpret what this behaviour suggests about the individual's culture (Tilley, 2015). Stage 1 involves describing the pathology of the skeletal remains and examining the corresponding lifeways context of the individual. Stage 2 describes the possible impacts of the pathology on the individual's life and ability to contribute to the social group. This stage also identifies whether the care provided was in the form of direct support or accommodation of differences (Tilley, 2012). Stage 3 involves the creation of a basic model of care that the individual likely received within the context of their culture. Stage 4 concludes with an interpretation of the model of care and infers what it suggests about the social practices, beliefs and values of the individual's social group (Tilley, 2012).

The bioarchaeology of care model can be used to examine the presence of complex behaviour in extinct hominids such as Neanderthals (Homo neanderthalensis). Neanderthals evolved in Europe around 300,000 years ago and spread into North Africa, the Middle East and Central Asia before disappearing from the fossil record around 25,000 years ago (Solecki, 1971). The discovery of another species of hominids that coexisted with anatomically modern Homo sapiens for thousands of years sparked a debate about whether the cognitive and behavioural complexity of Neanderthals matched humans (D'Errico et al., 2003). Investigating the presence of caregiving behaviour in Neanderthals contributes to this discussion of Neanderthal cognition and is useful for understanding the evolution of complex behaviour and emotions in hominids (Tilley, 2015). There have been seven cases where healthrelated care has been identified as necessary for the survival of the individual in Neanderthals (Tilley, 2015). For most of these cases, there has been a lack of comprehensive analysis into what caregiving may involve and what this behaviour suggests about the nature of Neanderthal society and cognition (Tilley, 2015).

The aim of this article is to examine health-related care for the Neanderthal Shanidar 1 through the model of bioarchaeology of care developed by Tilley (2012). Previous investigations into Shanidar 1 have noted the likelihood of care, but I was unable to find any thorough analysis of the type of care received and what caregiving suggests about the Neanderthals in the region. This article will address the context of Shanidar 1 before examining the evidence of health-related care using the four stages 
in the bioarchaeology of care methodology as a guide for identifying the type of care experienced by the individual and infer what this means for our understanding of Neanderthal behaviour and culture.

\section{Lifeways context of Shanidar Neanderthals}

The Shanidar Cave site was first discovered in 1951 by Ralph Solecki and is located in the Zagros mountain range in the Kurdistan Region of Northern Iraq (Solecki, 1971). The site contained the partial skeletons of nine Neanderthals with varying ranges of preservation and completeness (Solecki, 1971). The remains were not part of a single deposit, suggesting the area may have been used as a seasonal shelter home for the groups in the area (Trinkaus \& Zimmerman, 1982). The Neanderthals at Shanidar probably lived in small, kin-based, mixed-age groups that were highly mobile and frequently travelled between settlements in the region (Pettitt, 2000). Biologically, they resembled other Neanderthals discovered in Europe and had a more robust skeleton, more well-developed muscle attachment sites, greater weight, smaller height, lower sexual dimorphism and higher levels of developmental stress compared to anatomically modern humans in the region (Davies \& Underdown, 2006). The Shanidar Neanderthals were probably skilled, strategic hunters that had a knowledge of fire and subsisted on a diet of mainly meat, as evidenced by the bones of goats, sheep and cattle that were found at the site (Solecki, 1971). Recently, new evidence of starch granules embedded in the dental calculus of teeth from Shanidar suggest they also exploited plants as an alternative food source (Hardy, 2012). Neanderthals manufactured stone tools and weapons that belonged to the Mousterian culture, which were found in association with the remains at Shanidar (Solecki, 1971). The material culture and skeletal remains resemble those found in Europe, despite the geographic distance suggesting there was little change in Neanderthal society over time and space. The Neanderthals at Shanidar had a high frequency of degenerative joint disease, osteoarthritis and antemortem trauma that is indicated by bone deposition and remodelling associated with healing (Trinkaus \& Zimmerman, 1982). The high degree of trauma may be related to occupational hunting, interpersonal violence or preservation and sample bias. The skeletal remains at Shanidar were damaged by rockfalls, complicating the estimation of health-related care due to the fragmentary nature of the remains and the high degree of postmortem damage.

\section{Pathology of Shanidar 1}

Shanidar 1 is the most complete skeleton found from the Shanidar deposit and represents an adult male Neanderthal that dates from between 45,000 and 50,000 years ago (Solecki, 1971). He suffered from multiple potentially debilitating 
pathologies and was between the ages of 35 and 50 at time of death, which is considered an advanced age for a Neanderthal (Trinkaus \& Zimmerman, 1982). The skull of Shanidar 1 provides evidence of healed scars on the right frontal bone and a crushing fracture to the left orbit, frontal bone and zygomatic bone. This fracture was healed but permanently deformed the individual's left upper face and may have resulted in blindness in the left eye and damage to the frontal lobe and cerebral motor cortex (Trinkaus \& Zimmerman, 1982). The right clavicle, scapula and humerus of Shanidar 1 were smaller, thinner and less developed compared to the bones in the left arm due to a possible nerve injury or paralysis of the right arm from an early age (Trinkaus \& Zimmerman, 1982). The right humerus also has evidence of two healed fractures, with one on the olecranon fossa that may represent amputation of the lower arm prior to death. There was no evidence of the right radius, ulna or hand bones found at the site, which supports this idea of an antemortem amputation (Solecki, 1971).

The right metatarsals of Shanidar 1 have a healed fracture that was probably the result of direct trauma to the right foot or leg that is associated with degenerative joint disease around the articulation of the talus, calcaneus, fibula and tibia bones (Trinkaus \& Zimmerman, 1982). The right patella also has degenerative joint disease and the formation of exostoses. The left tibial diaphysis is slightly abnormally curved compared to the right tibia, which may have been caused by trauma or as the result of a change in weight bearing due to other injuries (Trinkaus \& Zimmerman, 1982). It has been suggested that Shanidar 1 may have also suffered from diffuse idiopathic skeletal hyperostosis (DISH), which has been used to explain bony outgrowths on the vertebra and ligament attachment sites (Crubezy \& Trinkaus, 1992). The order and timing of the injuries cannot be determined, so it is unknown which one was the primary injury or how long Shanidar 1 survived with the pathologies (Trinkaus \& Zimmerman, 1982). The remains were damaged by a rockfall and it is unknown whether this was related to cause of death or occurred after the individual had been buried in the cave.

\section{Impact of pathology}

Shanidar 1 suffered from various trauma-related pathologies that would have caused a variety of symptoms and negatively impacted his ability to function within his social group. Shanidar 1 experienced trauma to his left orbit and frontal bone that resulted in either permanent or temporary blindness, which would have made it difficult to hunt large mammals (Solecki, 1971). This skull fracture was probably associated with swelling, pain or bruising at the site, and may have resulted in headaches, nausea and confusion (Ellis, 2015). The trauma may have also resulted in brain damage, particularly to the left frontal lobe that controls motor function, emotional response, speech, reasoning and problem solving (Trinkaus \& Zimmerman, 1982). 
If Shanidar 1 experienced damage to his left frontal lobe, he may have experienced a wide variety of symptoms including difficulty controlling fine motor function in the right side of his body, personality changes, impaired judgement, difficulty speaking and the inability to solve problems (Neuroskills, 2016). Any damage to the frontal lobe would have impacted Shanidar 1's life and ability to perform tasks within his social group. He may not have been able to successfully communicate verbally with his group and may have experienced weakness in the right side of his body, which would negatively affect his mobility and ability to hunt and make tools. It is possible the cranial trauma was the primary injury that may have indirectly resulted in the secondary injuries, particularly the paralysis of the right arm via damage to the motor cortex (Trinkaus \& Zimmerman, 1982). The paralysis and amputation of Shanidar 1's right arm meant he would have experienced difficulty performing daily tasks and would not have been able to contribute to the main subsistence activity of hunting and preparing food or making weapons for the group (Davies \& Underdown, 2006). Shanidar 1 could have compensated for his ineffective right arm by developing his left arm to perform tasks or using his teeth as additional tools so he was able to contribute to his social group.

Shanidar 1 suffered from degenerative joint disease in his right foot and knee that resulted in the formation of bony spurs in the areas where the bones rub together (ACFAS, 2016). The condition would have resulted in pain, inflammation and stiffness in his ankle and knee that would have limited movement and would have been aggravated when he placed weight or pressure on the affected joints (ACFAS, 2016). Walking over long distances would have been difficult and painful for Shanidar 1 and may have affected his ability to perform everyday tasks, contribute to subsistence activities or keep up with the social group when travelling between settlements. Shanidar 1 may have also suffered from DISH, which may have caused additional pain, stiffness and restricted range of motion in the affected areas (Mayo Clinic, 2015). Shanidar 1 suffered from a wide range of pathologies and would have required health-related care in order to survive to his age at death.

\section{Model of care}

Shanidar 1 required health-related care in the form of direct support and accommodation at various points in his life. Shanidar 1 suffered from multiple fractures that would have required treatment and, most importantly, rest, in order to heal (Trinkaus \& Zimmerman, 1982). It would have been necessary for his social group to understand his reduced role in hunting and mobility while he recovered. If the cranial trauma had resulted in brain damage he may have required help with movement and protection from hazards while he recovered from the initial injury. Any damage to the frontal cortex would have required his social group to understand and accept his change in personality, potential lack of speech and impaired judgement. 
They would have been required to monitor his behaviour to ensure he did not place himself in danger due to loss of inhibitions and problem solving skills. Healthrelated care for Shanidar 1 may have also caused the amputation of his lower right arm (Trinkaus \& Zimmerman, 1982). If his arm was trapped or severely damaged by trauma or infection, members of his social group may have intentionally removed his arm in order save his life. In this situation, the amputation of his arm could be seen as a type of surgery and be classified as a successful treatment due to his survival. It remains unknown whether the amputation was intentional or accidental but if it was intentional it suggests the Shanidar Neanderthals had a knowledge of how seal off blood vessels to control bleeding (Clements, 2008).

Shanidar 1 may have needed care for degenerative joint disease in the form of physical therapy and massage to strengthen the muscles or the application of heat to relieve pain and stiffness or cold to reduce inflammation (Pain Medicine Consultants, 2016). Shanidar 1 may have required assistance in moving between campsites and to change posture, and would have benefited from pain relief in the form of medicinal plants. There is pollen evidence of medicinal plants in association with Shanidar 4, another burial at the site, that could have been used as pain relief but it is unknown whether these properties were known to the Neanderthals (Solecki, 1971). There is currently not enough evidence to suggest Neanderthals actively exploited medicinal plants, but this does not mean they were unaware of the therapeutic benefits of certain plants (Hardy, 2012). Shanidar 1 may have needed help maintaining hygiene and stretching affected muscles, and needed protection from hazards. It would have to have been accepted by his social group that Shanidar 1 was unable to hunt and probably had mobility issues, but he would still need to be actively included within the group in order to maintain mental health (Tilley, 2015). Shanidar 1 may have contributed to his social group in other ways instead of hunting, for example he may have been a teacher of knowledge or skills, or he may have been an additional caregiver of infants and younger members of the social group.

\section{Interpretation and implications for understanding Neanderthals}

Shanidar 1 suffered from a high incidence of trauma and died at an advanced age, suggesting he was provided with health-related care throughout his life. The survival of Shanidar 1 to an old age implies Neanderthals that were disabled or diseased were still accepted and cared for by the social group (Solecki, 1971). This contrasts with earlier views of Neanderthals that proposed trauma diminished the value of an individual, and disabled individuals were more likely to be abandoned by their social group (Pettitt, 2000). Caregiving could suggest Neanderthals had a high level of social and emotional complexity that reflected the principles of compassion and empathy for other individuals within their social group. This behaviour may 
have evolved due to the cooperative nature of hominid social groups, or it could be an extension of kinship-based altruism as Neanderthals mainly lived in small, closely related groups (Davies \& Underdown, 2006). Cooperation would have been incredibly important for the survival of the group, and flexibility in behaviour and roles within the group would be necessary to ensure survival. Care may have also evolved as an empathic response or reciprocal altruism where members of the group helped others to ensure they would receive care if it was required in the future (De Waal, 2008). The presence of health-related care in Neanderthals also suggests caregiving behaviour is not unique to Homo sapiens and may have evolved in an early hominid ancestor to both humans and Neanderthals (Hublin, 2009).

The level of care provided to Shanidar 1 suggests the Neanderthals in the region lived in cooperative kinship groups where caring for an injured individual was performed despite the social and economic cost to the group. This may suggest that the cost of caring for a disabled individual in the group was outweighed by kinship ties or possible benefits the person could provide by being a teacher. Caregiving behaviour reflects acceptance of a disabled individual within a social group and highlights the behavioural flexibility of the Shanidar Neanderthals (Solecki, 1971). The presence of health-related care can also provide information on the personality of the person receiving care. The survival of Shanidar 1 with multiple pathologies suggests he was resilient and determined and the ongoing care he received may reflect his continued important status within the social group. It is impossible to determine whether the care received by Shanidar 1 was a typical response of Neanderthals towards a disabled member of the group or whether the care he received was due to his status, skills or knowledge that was necessary for group survival (Tilley, 2015). The survival of Shanidar 1 with multiple pathologies suggests caregiving behaviour was present in his social group and may indicate compassion, cooperation and acceptance of people with a disability.

Further investigations into the Shanidar Neanderthals, including Shanidar 1, have been hindered by the political and social upheaval in Iraq that has prevented further investigations of the site. The instability in the region has led to the disappearance of all the Shanidar remains except Shanidar 3, which was transported to America for study (Trinkaus, 1982). Bioarchaeologists investigating the Shanidar Neanderthals have had to use cast models of the bones, which may not include all the available information and could affect the interpretation of the remains. Hopefully, stability will return to the region so that archaeologists can continue to excavate the region in the hope of discovering more fossil remains that may lead to a greater understanding of the biology, behaviour and intelligence of Neanderthals in the region. 


\section{Conclusion}

The bioarchaeology of care methodology is a useful tool for examining healthrelated care in extinct hominids such as Neanderthals. One such Neanderthal, Shanidar 1, survived to an old age with multiple potentially debilitating pathologies, which suggests he was provided with health-related care at various points in his life. Shanidar 1 was provided with care in the form of both direct support and accommodation of his changed role within the social group. The evidence of healthrelated care in relation to Shanidar 1 can be used to interpret Neanderthal society, and suggests the Neanderthals were willing to change their behaviour to accommodate an injured and disabled individual. The caregiving behaviour also suggests Shanidar 1 was accepted and included within his social group despite his diminished ability to contribute to the economic needs of the community. The evaluation of care in Shanidar 1 can be used to understand the presence and evolution of complex behaviour and emotions in Neanderthals.

\section{Bibliography}

American College of Foot and Ankle Surgeons (ACFAS). (2016). Osteoarthritis of the Foot and Ankle. Retrieved from www.foothealthfacts.org/footankleinfo/ osteoarthritis.htm (accessed April 2016).

Clements, I. (2008). How Amputation Works. Retrived from health.howstuffworks. $\mathrm{com} /$ medicine/modern-treatments/amputation.htm (accessed April 2016).

Crubezy, E. \& Trinkaus, E. (1992). 'Shanidar 1: A Case of Hyperostotic Disease (DISH) in the Middle Paleolithic'. American Journal of Physical Anthropology, 89, 411-420. doi.org/10.1002/ajpa.1330890402

Davies, R. \& Underdown, S. (2006). 'The Neanderthals: A Social Synthesis'. Cambridge Archaeological Journal, 16(2), 145-164. doi.org/10.1017/ S0959774306000096

D’Errico, F., Henshilwood, C., Lawson, G., Vanhaeren, M., Tillier, A., Soressi, M., Bresson, F., Maureille, B., Nowell, A., Lakarra, J., Backwell, L. \& Jul, M. (2003). 'Archaeological Evidence for the Emergence of Language, Symbolism and Music-An Alternative Multidisciplinary Perspective'. Journal of World Prehistory, 17(1), 1-70. doi.org/10.1023/A:1023980201043

De Waal, F. (2008). 'Putting the Altruism Back into Altruism: The Evolution of Empathy'. Annual Reviews Psychology, 59, 279-300. doi.org/10.1146/annurev. psych.59.103006.093625 
Ellis, M. (2015). 'Skull Fractures'. Healthline. Retrieved from www.healthline.com/health/skull-fracture\#Overview1 (accessed April 2016).

Hardy, K (2012). 'Neanderthal Medics? Evidence for Food, Cooking and Medicinal Plants Entrapped in Dental Calculus'. Naturwissenschaften, 99, 617-626. doi. org/10.1007/s00114-012-0942-0

Hublin, J. (2009). 'The Prehistory of Compassion'. Proceedings of the National Academy of Science, 106(16), 6429-6430. doi.org/10.1073/pnas.0902614106

Mayo Clinic. (2015). Diffuse Idiopathic Skeletal Hyperostosis (DISH). Mayo Foundation for Medical Education and Research. Retrieved from www. mayoclinic.org/diseases-conditions/diffuse-idiopathic-skeletal-hyperostosis/ basics/definition/con-20024713 (accessed April 2016).

Neuroskills. (2016). Traumatic Brain Injury Resource Guide-Frontal Lobes. Centre for Neuroskills, USA. Retrieved from www.neuroskills.com/brain-injury/frontallobes.php (accessed April 2016).

Pain Medicine Consultants. (2016). Osteoarthritis Pain Management. Pain Medicine Consultants, USA. Retrieved from www.painmedicineconsultants. com/conditions-osteoarthritis-orthopedics-walnut-creek-ca.html (accessed April 2016).

Pettitt, P. (2000). 'Neanderthal lifecycles: Developmental and Social Phases in the Lives of the Last Archaics'. World Archaeology, 31(3), 351- 366. doi.org/ $10.1080 / 00438240009696926$

Solecki, R. (1971). Shanidar: The First Flower People. New York: Alfred A. Knophf.

Tilley, L. (2012). 'The Bioarchaeology of Care'. The SAA Archaeological Record: New Directions in Bioarchaeology, Part II, 12, 39-41.

Tilley, L. (2015). Theory and Practice in the Bioarchaeology of Care. Springer. doi. org/10.1007/978-3-319-18860-7

Tilley, L. \& Oxenham, M. (2011). 'Survival Against the Odds: Modelling the Social Implications of Care Provision to Seriously Disabled Individuals'. International Journal of Paleopathology, 1, 35-42. doi.org/10.1016/j.ijpp.2011.02.003

Trinkaus, E. (1982). 'Shanidar 3 Neandertal'. American Journal of Physical Anthropology, 57, 37-60. doi.org/10.1002/ajpa.1330570107

Trinkaus, E. \& Zimmerman, M. (1982). 'Trauma Among the Shanidar Neandertals', American Journal of Physical Anthropology, 57, 61-76. doi.org/10.1002/ ajpa.1330570108 
This text is taken from The ANU Undergraduate Research Journal, Volume Eight, 2016, edited by Daniel McKay, published 2017 by ANU eView, The Australian National University, Canberra, Australia.

dx.doi.org/10.22459/AURJ.08.2016.07 